\title{
Small-Time Local Controllability of a Differential Drive Robot with a Limited Sensor for Landmark-Based Navigation
}

\author{
Rafael Murrieta-Cid and Jean-Bernard Hayet \\ Centro de Investigación en Matemáticas, CIMAT \\ Guanajuato, México \\ \{murrieta, jbhayet\}@cimat.mx
}

\begin{abstract}
This work studies the interaction of the nonholonomic and visibility constraints of a robot to maintain visibility of a landmark. The robot is a differential drive system (nonholonomic robot) and has a sensor with limited capabilities (limited field of view). In this research, we want to determine whether or not a robot can always maintain visibility of a landmark during the execution of a path between any two locations. We present two kinematic models. First, a robot with 3 controls, where the controls correspond to the two wheels velocities plus one independent controlled sensor. Second, a model with only 2 controls, which controls both the wheels and the sensor rotation. We show that our system (with 3 or 2 controls) is small-time local controllable.
\end{abstract}

\section{Introduction}

Landmarks have been frequently used in robotics, either to localize the robot with respect to them [221] or to navigate 14/17/4/8. In robot navigation, a landmark can be used as a goal or sub-goal that the robot must reach or perceive during the robot motion. A landmark can be defined in several manners: From a set of points in an image having useful properties (i.e. saliency), up to a $3 \mathrm{D}$ object associated with a semantic label and having 3-D position accuracy.

To use landmarks in the context of mobile robotics, the first basic requirement is to perceive them during the robot motion. Even though, landmarks have been extensible used in robotics [5|2|21|14|4, to our knowledge this is the first attempt to show whether or not a robot under the interaction of its nonholonomic and visibility constraints is small time local controllable. We believe that our research is very pertinent given that a lot of mobile robots are nonholonomic systems equipped with limited field of view sensors (lasers or cameras).

Our final goal is to show that if a collision free path between any two locations exists for an unconstrained system (i.e. a holonomic robot equipped with omnidirectional field of view sensor) to maintain landmark visibility, then a feasible path shall exist for our system under their motion and perception constraints.

A. Gelbukh and A.F. Kuri Morales (Eds.): MICAI 2007, LNAI 4827, pp. 800 8102007.

(C) Springer-Verlag Berlin Heidelberg 2007 
In other words, we want to determine whether or not a constrained robot can always maintain visibility of a landmark during the execution of a path between any two locations. To do so, we need to determine whether or not the system is small time locally controllable. This is the main motivation of this work.

Small time local controllability (STLC) implies that a system can locally maneuver in any direction. Let us define $\mathcal{M}$ as a manifold; if the system is STLC at all $x \in \mathcal{M}$, then the system can follow any curve on $\mathcal{M}$ arbitrarily closely. This has an important implication in environments with obstacles or sensor constraints, as a STLC system can always maneuver through clutter space, since any motion of a system with no motion constraints can be approximated by a motion constrained system that is STLC anywhere [116.

In 3 we have shown that a differential drive robot (DDR) with field-ofview and range constraints is controllable. We have also provided the shortest paths in the sense of distance for the field-of-view constraint (without the range constraint). However, in that work we have not shown whether or not the system is small time locally controllable. STLC implies controllability but the converse property is not true. The difference between controllability and STLC can be described with an intuitive example (a definition is given in section 2.1), a car that can only go forward (Dubins car [7]) is controllable, but it is not small time local controllable. Such a car will need a long motion to reach a close position behind it. Note that such a long motion may produce robot collision (in an environment with obstacles) or force the robot to lose landmark visibility by moving outside the sensor limits.

The research reported in this paper differs from our previous efforts in the following main point: We shall present an existence proof of small time-local controllability of the system under its motion and perception constraints. We will present two robot models. First, a robot with 3 controls, where the controls correspond to the two wheel velocities plus one independent controlled sensor. Second, a robot model with 2 controls. In this last model, the sensor is a slave of the robot's wheels, thus, the sensor moves in function of the wheels' motors. The model with 2 controls assumes that the robot is moving along the optimal paths (composed by straight lines and sectors of spirals) presented in [3], as the visibility constraint is satisfy exactly at each point.

Problem definition: The initial and final locations and the path can be given to the robot as input or be computed by a planner. The initial and final configurations satisfy the visibility constraint. The robot is a differential drive system (nonholonomic robot). The robot is equipped with a limited field of view sensor. The landmark is static and the robot observer must maintain the landmark within the sensor's field of view. Visibility is defined geometrically, i.e. the landmark is visible from the robot sensor if a clear line of sight can join them and the landmark is within the minimal and maximal sensor field of view. Thus, this work focuses on studying the interaction of the nonholonomic and visibility constraints of the robot observer. More precisely, the problem to solve here consists in proving whether or not this system is small time locally controllable under its kinematic and perceptual constraints. 


\section{Nonholonomic Constraints}

Nonholonomic systems are characterized by constraint equations involving the time derivatives of the system configuration variables. In a nonholonomic system these equations are not integrable; in fact this property corresponds to the core of the definition of a nonholonomic system. The state transition equation of a system $\dot{X}=f(X, U)$, where $X$ is the state vector, indicates how the state of the system changes over the time according to some inputs. If the state transition equation is integrable, it is said that the corresponding system is holonomic; otherwise, the system is nonholonomic (Frobenius theorem, see below).

Typically, non-integrable equations arise when the system has less controls than configuration variables. For instance, a differential drive robot has two controls (right and left wheel velocities) while moving in a 3-dimensional configuration space $X=(x, y, \theta)^{T}$.

From the point of view of motion planning, the main implication of nonholomic constraints is that any collision-free path in the configuration space does not necessarily correspond to a feasible path for the system. Purely geometric techniques to find collision-free paths do not apply directly to nonholonomic systems.

Motion planning with nonholonomic constraints has been a very active research field (a nice overview is given in [12]). The most important results in this field have been obtained by addressing the problem with tools from differential geometry and control theory. Laumond pioneered this research [9] and produced the result that a free path for a free-flying robot moving among obstacles in a $2 \mathrm{~d}$ work-space can always be transformed into a free path for a nonholonomic car-like robot by making car maneuvers [11. Sussman and Liu [20] propose an algorithm for constructing a sequence of admissible trajectories in the presence of obstacles for nonholonomic systems. Li and Canny [15] apply controllability theory for non-linear systems to nonholonomic robots. Murray and Sastry [16] use sinusoidal control in steering nonholonomic systems.

The study of optimal paths for nonholonomic systems has also been an active research topic. Dubins [7] determined the shortest paths for a car-like robot than can only go forward. Reed and Shepp extended this work and established the shortest length paths for a car-like robot that can move forward and backward [19. In [18 a complete characterization of the shortest path for a car-like robot is given.

Balkcom and Mason determined the time-optimal trajectories for a differential drive robot [1]. These trajectories for a differential drive robot were found using Pontryagin's Maximum Principle and geometric analysis. All these results about optimal paths assume that the nonholonomic robot moves in the free space (without obstacles).

\subsection{Integrability and Controllability}

The Frobenius and Chow theorems are used to establish integrability and controllability. 
A system is said to be nonholonomic if the state transition equation is not integrable. Frobenius theorem establishes that the state transition equation is integrable if and only if all the vectors fields that can be obtained by the Lie bracket operations are contained in $\Delta$.

$\Delta$ is called distribution and can be considered as a vector space. $\Delta=$ $\operatorname{span}\left(\alpha^{1}(x), \alpha^{2}(x), \ldots, \alpha^{n}(x)\right)$ is the set of vector fields $\alpha^{i}, \alpha^{i}(x)$ is a vectorvalued function of $x$.

Two important properties to be determined in a nonholonimic system are controllability and small time local controllability (STLC).

Controllability means that the nonholonomic robot is able to overcome its differential constraints by using velocities that are not directly permitted by the state transition equation. That implies that any state can be reached from any other state.

If a system is STLC then the system can move in any direction, in an arbitrarily small amount of time. More formally, let $R(x, \delta t)$ denotes the set of all points reachable in time $\delta t$. A system is small-time locally controllable if for all $x \in X$ and any $\delta t>0, x$ lies within an open set contained by $R(x, \delta t)[13$. These new velocities not directly permitted in the state transition equation are generated with the Lie Bracket.

The $i^{\text {th }}$ component of the Lie bracket is given by:

$$
Z_{i}=\sum_{j=1}^{n}\left(P_{j} \frac{\partial Q_{i}}{\partial x_{j}}-Q_{j} \frac{\partial P_{i}}{\partial x_{j}}\right)
$$

where $P_{j}$ and $Q_{j}$ are vector fields.

Chow's theorem says that a system is small-time controllable if and only if the dimension of $C L A(\Delta)$ is the dimension of $X$ and the system is symmetric [6], where $C L A$ is the Control Lie Algebra, $C L A(\Delta)$ is the set of of vector fields $\alpha^{i}$ and all the new linearly-independent vectors that can be generated from the Lie bracket operations by using the control inputs. $X$ is the robot state (velocities that can be applied to the configuration space variables). Note that the dimension of $C L A(\Delta)$ can never be greater than the dimension of robot state $X$.

\section{$3 \quad$ Visibility Constraints}

In the problem addressed in this work, a differential drive robot must maintain visibility of a static landmark. The robot is equipped with a sensor that has a limited field of view. It is assumed that the robot is equipped with a pan controllable sensor.

We make the usual assignment of body attached frame to the robot. The origin is at the midpoint between the two wheels, $y$-axis parallel to the axle, and the $x$-axis pointing forward, parallel to the robot's heading. $\theta$ is the angle from the world $x$-axis to the robot $x$-axis. The robot can move forward and backward, 
the heading of the robot is defined as the direction in which the robots moves, so the heading angle with respect to the robot's $x$-axis is zero or $\pi$.

The Landmark is at the origin of the coordinate system. The position of the robot with respect to the landmark is defined in polar coordinates by the following equations:

$$
\begin{aligned}
& r=\sqrt{x^{2}+y^{2}} \\
& \alpha=\arctan \frac{y}{x}
\end{aligned}
$$

The sensor is placed so that the optical center lies directly above the origin of the robot's local coordinate frame. The sensor pan angle $\phi$ is the angle from the robot's $x$-axis to the optical axis. The range of the sensor rotation is limited, such that $\phi \in\left[\phi_{1}, \phi_{2}\right]$. Figures 1 and 2 show these conventions.

\section{Proving that the System is Small Time Local Controllable}

We present two kinematic models. First, a robot with 3 controls, where the controls correspond to the two wheel velocities plus one independent controlled sensor. Second, a model with only 2 controls, where those inputs control both the wheels and the sensor rotation.

\subsection{Differential Drive Robot with 3 Controls}

In this case the system constraints are the following:

1. The system is a differential drive robot (nonholonomic system) that can move forward and backward, hence it is a symmetric system.

2. The landmark is always within the sensor field of view, i.e.,

$$
(\alpha+\pi)-\theta-\epsilon \leq \phi \leq(\alpha+\pi)-\theta+\epsilon
$$

where $\epsilon>0$ represents the sensor half field of view (See figure 1).

3. The sensor pan angle is limited such that $\phi \in\left[\phi_{1}, \phi_{2}\right]$. The sensor is pointed forward $0 \in\left[\phi_{1}, \phi_{2}\right]$, and hence the robot can directly approach the landmark.

The robot configuration is given by $X=(x, y, \theta, \phi)$ or equivalently by polar coordinates $(r, \alpha, \theta, \phi)$. Note that $\phi$ defines the sensor orientation with respect to the robot heading; the sensor does not need to be directly pointing to the landmark, but the landmark must be within the sensor's field of view. This setting gives flexibility with respect to sensor heading and hence is tolerant to control errors. However, to define this model 4 configuration variables and 3 controls are needed. 


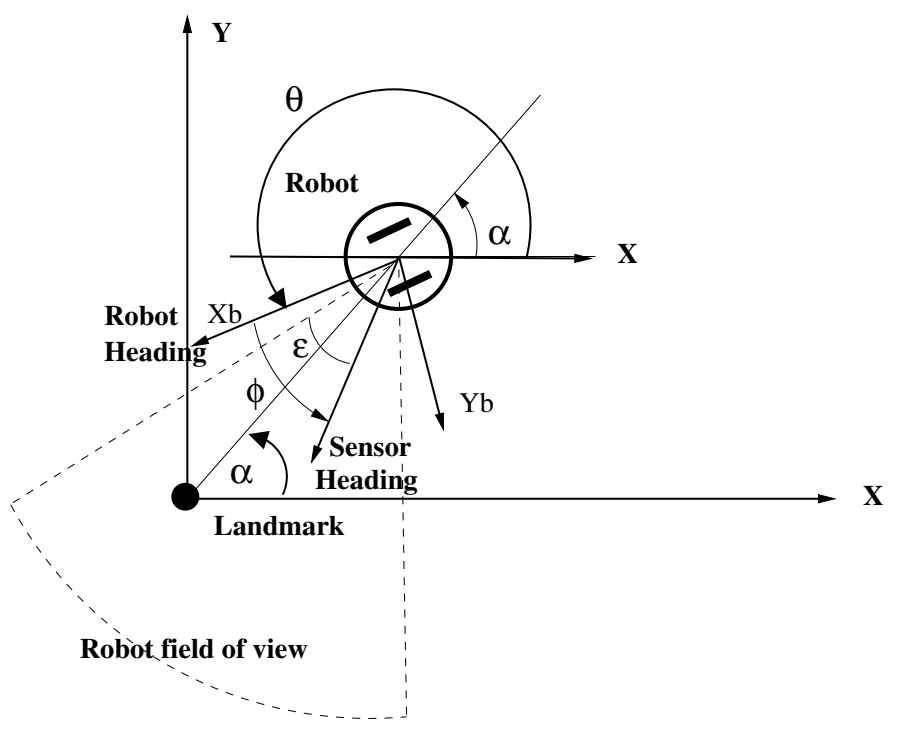

Fig. 1. Differential drive robot with 3 controls

In this case, the state transition equation for our differential drive robot equipped with a controllable sensor can be expressed as:

$$
\left(\begin{array}{c}
\dot{x} \\
\dot{y} \\
\dot{\theta} \\
\dot{\phi}
\end{array}\right)=\left(\begin{array}{ccc}
\cos \theta & 0 & 0 \\
\sin \theta & 0 & 0 \\
0 & 1 & 0 \\
0 & 0 & 1
\end{array}\right)\left(\begin{array}{l}
\mu_{1} \\
\mu_{2} \\
\mu_{3}
\end{array}\right)
$$

The controls of our system are $\mu_{1}, \mu_{2}$ and $\mu_{3}$. Controls $\mu_{1}, \mu_{2}$ are the same than in the differential drive robot without the sensor, where $\mu_{1}=w_{r}+w_{l}$ and $\mu_{2}=w_{r}-w_{l} . w_{r}$ and $w_{l}$ are the angular velocities of the left and right wheels respectively. Control $\mu_{1}$ means going in a straight-line motion and control $\mu_{2}$ corresponds to a rotation centered on the robot.

A new control must be added to the system given that the sensor is moved by an independent motor. This new control is $\mu_{3}=w_{c}$, where $w_{c}$ is the sensor angular velocity. Control $\mu_{3}=1$ corresponds to a sensor rotation.

Chow's theorem is used in order to prove that the system is STLC (see section 2). The first step to know the dimension of $C L A(\Delta)$ is to compute the Lie brackets of all the original vectors fields. The original vector fields are: $\vec{V}=$

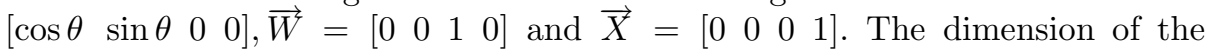
distribution $\Delta$ of this system is 3 and the dimension of state $X$ is 4 .

The possible vector fields combinations are: $[\vec{V}, \vec{W}],[\vec{V}, \vec{X}],[\vec{W}, \vec{X}]$.

The resulting components of the Lie bracket for the different vector field combinations are: $\vec{Z}=[\vec{V}, \vec{W}]=[\sin \theta-\cos \theta \quad 00]$, Lie bracket for the other combinations are null vectors. 
$\vec{Z}$ is linearly independent from $\vec{V}, \vec{W}$ and $\vec{X}$. The determinant of the matrix A:

$$
A=\left(\begin{array}{cccc}
\cos \theta & \sin \theta & 0 & 0 \\
0 & 0 & 1 & 0 \\
0 & 0 & 0 & 1 \\
\sin \theta & -\cos \theta & 0 & 0
\end{array}\right) ; \quad\left|\begin{array}{cccc}
\cos \theta & \sin \theta & 0 & 0 \\
0 & 0 & 1 & 0 \\
0 & 0 & 0 & 1 \\
\sin \theta & -\cos \theta & 0 & 0
\end{array}\right| \neq 0
$$

is nonzero everywhere in the configuration space. Thus, the dimension of the $C L A(\Delta)$ is equal to the dimension of the system state $X$. Both of them are 4 . Therefore, the system is nonholonomic and small time local controllable.

\subsection{Differential Drive Robot with 2 Controls}

It is also possible to generate a state transition equation with just two controls. In this scheme, the sensor is pointing to the landmark according to the wheels velocities, thus the sensor control is not needed. As before, it must also be determined, whether or not this system is STLC.

In this case the constraints (1) and (3) are the same that in the case of a system with 3 controls. However, constraint (2) is stronger.

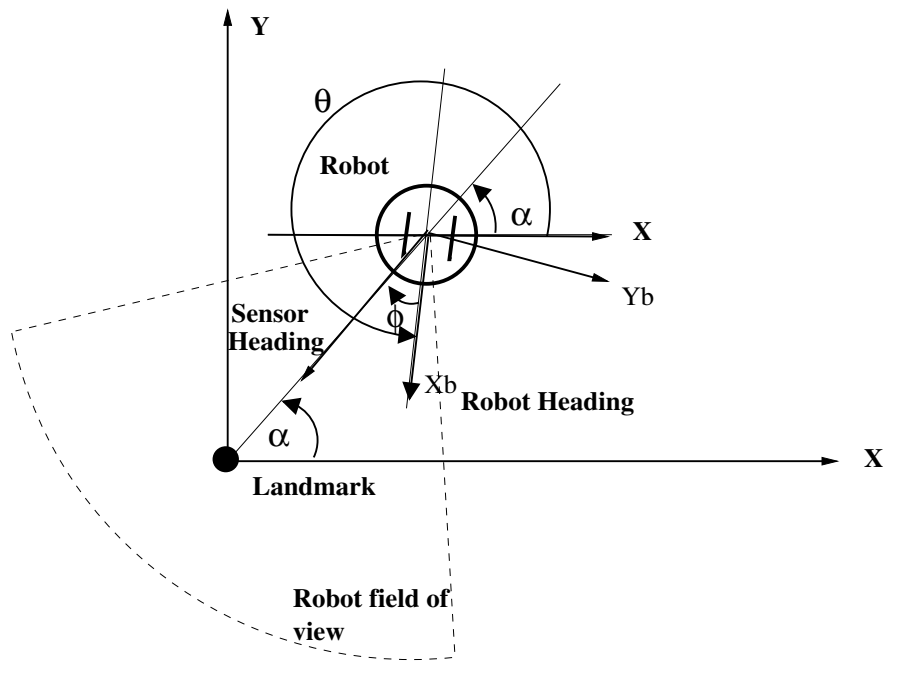

Fig. 2. Differential drive robot with 2 controls

The sensor optical axis is always pointing toward the landmark (see figure 2), so that :

$$
\theta=\alpha-\phi+\pi \text {. }
$$

The construction of the state transition equation is as follows. First, we get the derivative of equation 7 ;

$$
\dot{\phi}=\dot{\alpha}-\dot{\theta} .
$$


Then we obtain the derivative of equation 3 .

$$
\dot{\alpha}=\frac{1}{1+\frac{y^{2}}{x^{2}}} \frac{\dot{y} x-\dot{x} y}{x^{2}}=\frac{\dot{y} x-\dot{x} y}{x^{2}+y^{2}} .
$$

The controls are:

$$
\begin{aligned}
& \mu_{1}=w_{r}+w_{l} \\
& \mu_{2}=w_{r}-w_{l}
\end{aligned}
$$

Therefore, the

$$
\begin{aligned}
& \dot{\theta}=w_{r}-w_{l} \\
& \dot{x}=\cos \theta\left(w_{r}+w_{l}\right) \\
& \dot{y}=\sin \theta\left(w_{r}+w_{l}\right)
\end{aligned}
$$

The key observation is the following: $\phi$ is not a degree of freedom any more. It can be expressed as a function of $x, y$ and $\theta$. Hence, the robot configuration is totally defined by $(x, y, \theta) . \phi$ and $\dot{\phi}$ are constraints that the system must satisfy to maintain landmark visibility.

$\dot{\phi}$ can be expressed directly in function of the controls $\mu_{1}, \mu_{2}$ and the configuration variables $(\theta, x, y)$. This can be done by substituting in 8 , the values of $\dot{\alpha}$ and $\dot{\theta}$ from equations 9 and 12 ,

$$
\dot{\phi}=\frac{(y \cos \theta-x \sin \theta) \mu_{1}}{x^{2}+y^{2}}-\mu_{2} .
$$

Hence, the state transition equation takes the form:

$$
\left(\begin{array}{c}
\dot{x} \\
\dot{y} \\
\dot{\theta}
\end{array}\right)=\left(\begin{array}{cc}
\cos \theta & 0 \\
\sin \theta & 0 \\
0 & 1
\end{array}\right)\left(\begin{array}{l}
\mu_{1} \\
\mu_{2}
\end{array}\right),
$$

which is exactly the same of the differential drive robot 113. Because of the visibility restrictions, the two only basic motions are straight lines and logarithmic spirals [3]. We have already seen that the vector field associated to the straight line is $(\cos \theta, \sin \theta, 0)^{T}$. Now let us express the vector field associated to the spirals. The equations of these curves are [3]:

$$
r=r_{0} e^{\left(\alpha_{0}-\alpha\right) / \tan \phi}
$$

where $r_{0}, \alpha_{0}$ and $\phi$ remain constant along these curves. From this equation, we can easily derive the corresponding vector field, as $x=r \cos \alpha$ and $y=r \sin \alpha$ :

$$
\vec{W}=\left(\begin{array}{c}
-y-\frac{x}{\tan \phi} \\
x-\frac{y}{\tan \phi} \\
1
\end{array}\right) \text {. }
$$


Now remember that $\phi$ is a constant on all spirals, but its value is constrained by the robot position, as $\phi=\alpha-\theta+\pi$. Moreover, as $\alpha=\arctan \frac{y}{x}$, we can write :

$$
\frac{1}{\tan \phi}=\frac{1}{\tan \left(\arctan \frac{y}{x}+\pi-\theta\right)}=\frac{1-\tan \left(\arctan \frac{y}{x}\right) \tan (\pi-\theta)}{\tan \left(\arctan \frac{y}{x}\right)+\tan (\pi-\theta)}=\frac{x+y \tan \theta}{y-x \tan \theta} .
$$

Note that this vector field is not defined for $y=x \tan \theta$, which corresponds to zones where the robot has to follow a straight line, in fact, as it is pointing to the landmark. The last equation is combined with Eq,18 to get:

$$
\vec{W}=\left(\begin{array}{c}
-\frac{x^{2}+y^{2}}{y-x \tan \theta} \\
-\tan \theta \frac{x^{2}+y^{2}}{y-x \tan \theta} \\
1
\end{array}\right) .
$$

The Lie bracket $\vec{Z}=[\vec{V}, \vec{W}]$ can be computed by using formula of Eq1, which leads to

$$
\vec{Z}=\left(\begin{array}{c}
\sin \theta-2 \frac{x \cos \theta+y \sin \theta}{y-x \tan \theta} \\
-\cos \theta-2 \tan \theta \frac{x \cos \theta+y \sin \theta}{y-x \tan \theta} \\
0
\end{array}\right) .
$$

Last step of our demonstration, the determinant of the matrix $B$ :

$$
\begin{aligned}
B & =(\vec{V}, \vec{W}, \vec{Z}) \\
& =\left(\begin{array}{ccc}
\cos \theta & -\frac{x^{2}+y^{2}}{y-x \tan \theta} & \sin \theta-2 \frac{x \cos \theta+y \sin \theta}{y-x \tan \theta} \\
\sin \theta & -\tan \theta \frac{x^{2}+y^{2}}{y-x \tan \theta} & -\cos \theta-2 \tan \theta \frac{x \cos \theta+y \sin \theta}{y-x \tan \theta} \\
0 & 1 & 0
\end{array}\right)
\end{aligned}
$$

is +1 for all values of $x, y, \theta$. Hence, again this system is small time local controllable everywhere in the configuration space.

\section{Conclusion and Future Work}

It is possible to draw analogies between our system and the car-like robot. In the car-like robot the steering angle is constrained due to mechanical stops in the steering gear. This constraint forces the robot to move with curvature bounds [11]. In our system, these bounds also exist [3] due to the constraint of maintaining landmark visibility and the assumption that the sensor can rotate a limited angle.

In the car-like robot the curvature constraint is upper-bounded by the same value wherever it is defined [10. In our system the bound changes according to the orientation of the robot with respect to the landmark.

In this paper, we have shown that our systems (with 3 or 2 controls) are small-time local controllable. 
We are currently addressing the problem of landmark-based navigation in an environment with obstacles. Besides, the limitation in the sensor field of view induces the presence of a virtual obstacle in the configuration space. Therefore, it is necessary that there exists an open set around the robot to prove the existence of a path between any initial and final robot locations if one exists for an unconstrained system.

As in the case of a car-like system, the main difficulty in our system consists in determining the "size" of the free space allowed around the steering solution.

A way to compute this size is to determine the shortest paths for the system, this would allow us to determine a metric of the system. In [3] we have provided the shortest paths to maintain visibility of a landmark for a differential drive robot with field-of-view constraint. We plan to use those paths to determine lower and upper bounds of the paths' metric and the metric's induced topology.

The existence of obstacles (real or induced by sensor limitations) forces the use of some given metric in the plane. Given that the system is STLC, if the shortest-path metric induces the same topology as the metric used to measure the distance between the robot and the obstacles, then the existence of any "small" $\epsilon>0$ clearance between the robot and the obstacles in the plane would be enough to guarantee a feasible path.

\section{Acknowledgments}

This research was partially funded by CONACyT project 56754 .

\section{References}

1. Balkcom, D.J., Mason, M.T.: Time Optimal Trajectories for Differential Drive Vehicles. International Journal of Robotics Research 21(3), 199-217 (2002)

2. Betke, M., Gurvits, L.: Mobile Robot Localization using Landmarks. IEEE Transactions on Robotics and Automation 13(2), 251-263 (1997)

3. Bhattacharya, S., Murrieta-Cid, R., Hutchinson, S.: Optimal Paths for Landmarkbased Navigation by Differential Drive Vehicles with Field-of-View Constraints. IEEE Transactions on Robotics 23(1), 47-59 (2007)

4. Briggs, A.J., Detweiler, C., Scharstein, D., Vandenberg-Rodes, A.: Expected Shortest Paths for Landmark-Based Robot Navigation. International Journal of Robotics Research 12(8) (July 2004)

5. Bulata, H., Devy, M.: Incremental construction of landmark-based and topological model of indoor environments by a mobile robot. In: IEEE Int. Conf. on Robotics and Automation, IEEE Computer Society Press, Los Alamitos (1996)

6. Choset, H., Lynch, K.M., Hutchinson, S., Cantor, G., Burgard, W., Kavraky, L.E., Thrun, S.: Principles of Robot Motion: Theory, Algorithms, and Implementations. MIT Press, Cambridge (2005)

7. Dubins, L.E.: On curves of minimal length with a constraint on average curvature and with prescribed initial and terminal position and tangents. American Journal of Mathematics 79, 497-516 (1957)

8. Hayet, J.B., Lerasle, F., Devy, M.: A Visual Landmark Framework for Mobile Robot Navigation. Image and Vision Computing 25(8), 1341-1351 (2007) 
9. Laumond, J.P.: Feasible trajectories for mobile robots with kinematic and environment constraints. In: Hertzberger, L.O., Groen, F.C.A. (eds.) Intelligent Autonomous Systems, pp. 346-354. North-Holland, New York (1987)

10. Latombe, J.C.: Robot motion planning. Kluwer academic publishers, Dordrecht (1991)

11. Laumond, J.P., Jacobs, P.E., Taix, M., Murray, R.M.: A Motion planner for nonholonomic mobile robots. IEEE Trans. on Robotics and Automation 10(5), 577-593 (1994)

12. Laumond, J.P.: Robot Motion Planning and Control. Springer, Toulouse France (1998)

13. LaValle, S.M.: Planning Algorithms. Cambridge University Press, Cambridge (2006)

14. Lazanas, A., Latombe, J.-C.: Landmark-based robot navigation. Algorithmica 13, 472-501 (1995)

15. Li, Z., Canny, J.: Motion of two rigid bodies with rolling constraint. IEEE Trans. on Robotics and Automation 6, 62-72 (1990)

16. Murray, R.M., Sastry, S.S.: Nonholonomic motion planning: Steering using sinusoids. IEEE Trans. on Robotics and Automation 38(5), 700-716 (1993)

17. Murrieta-Cid, R., Parra, C., Devy, M.: Visual Navigation in Natural Environments: From Range and Color Data to a Landmark-based Model. Journal Autonomous Robots 13(2), 143-168 (2002)

18. Soueres, P., Laumond, J.P.: Shortest path synthesis for a car-like robot. IEEE Trans. on Autom Control 14(5), 672-688 (1996)

19. Reeds, J.A., Shepp, R.A.: Optimal Paths for a car that goes both forward and backwards. Pacific Journal of Mathematics 145(2), 367-393 (1990)

20. Sussmann, H.J., Liu, W.: Limits of highly oscillatory controls and the approximation of general paths by admissible trajectories, Tech. Rep. SYSCON-91-02., Rutgers Center for Systems and Control (February 1991)

21. Thrun, S.: Bayesian landmark learning for mobile robot localization. Machine Learning 33(1), 41-76 (1998) 